\title{
Impedance Model-Based Optimal Regulation on Force and Position of Bimanual Robots to Hold an Object
}

\author{
Darong Huang, ${ }^{1}$ Hong Zhan, ${ }^{1}$ and Chenguang Yang $\mathbb{D}^{2}$ \\ ${ }^{1}$ Key Laboratory of Autonomous Systems and Networked Control, School of Automation Science and Engineering, \\ South China University of Technology, Guangzhou 510641, China \\ ${ }^{2}$ Bristol Robotics Laboratory, University of the West of England, Bristol BS16 1QY, London, UK \\ Correspondence should be addressed to Chenguang Yang; cyang@ieee.org
}

Received 24 July 2020; Revised 3 September 2020; Accepted 24 October 2020; Published 17 November 2020

Academic Editor: Juan Sandoval

Copyright (c) 2020 Darong Huang et al. This is an open access article distributed under the Creative Commons Attribution License, which permits unrestricted use, distribution, and reproduction in any medium, provided the original work is properly cited.

\begin{abstract}
Bimanual robots have been studied for decades and regulation on internal force of the being held object by two manipulators becomes a research interest in recent years. In this paper, based on impedance model, a method to obtain the optimal target position for bimanual robots to hold an object is proposed. We introduce a cost function combining the errors of the force and the position and manage to minimize its value to gain the optimal coordinates for the robot end effectors (EE). To implement this method, two necessary algorithms are presented, which are the closed-loop inverse kinematics (CLIK) method to work out joint positions from desired EE pose and the generalized-momentum-based external force observer to measure the subjected force acting on the EE so as to properly compensate for the joint torques. To verify the effectiveness, practicality, and adaptivity of the proposed scheme, in the simulation, a bimanual robot system with three degrees of freedom (DOF) in every manipulator was constructed and employed to hold an object, where the results are satisfactory.
\end{abstract}

\section{Introduction}

Robots were invented mainly to help human being to carry out tasks, reducing the intensity of human labour, for example, grasping, holding, or moving an object. In such a process, we need robot to keep the object fixed on the end effector (EE) in a proper manner and move along the desired trajectory. Researchers have made effort to perfect these movements in many specific aspects. For instance, in [1], the optimal grasp rectangle for determining the correct position to pick up an object is obtained. Precision of robotic grasping is considered in [2], using probabilistic postgrasp displacement estimation method. Inspired by the scheme of human grasping, [3] presents a controller with tactile sensing and the grasp force can be adjusted as needed; for example, the object slips or not. In [4], the object with dynamics parameters and deformable shape is considered and the grasping forces can be regulated based on a similar tactile framework. The application scenarios of these motions vary from daily life (cloth grasping or towel folding [5]) to industry (plate and belt assembling [6]), military [7], and so on. The literature also demonstrates the fact of a trend of research on holding a target object by robots.

The above-mentioned implementations are related to managing to make an object "stick" to EE of one manipulator. These methods are good, but not better than multirobot system (MRS) to some extent [8]. Compared to a single manipulator, MRS is more complex but competent due to its higher redundancy, larger loading capability, and stronger robustness [9]. It has also been employed in many fields, for example, welding [10], assembly [11], and source seeking [12]. Generally, thanks to the aforementioned advantages, MRS is more prominent to handle complicated manipulations, such as curving and screwing. In the domain of MRS, the bimanual robotic system $[6,13]$ or the humanoid (dual-arm) robotic system $[14,15]$ has drawn a lot of attention from scientists and specialists.

In the specific domain of the bimanual robot system, the collaboration of the two manipulators becomes one of the research hotspots in the past decades [16-18]. The regulation 
of the internal force of a held object or the two robotic manipulators is a pronounced tendency and plenty of related methods appear. An adaptive global neural network control law for bimanual robots system is reported in [13] to simultaneously guarantee the internal forces to converge within a small neighbourhood of the desired values. Based on relative impedance models, in bimanual robots teleoperation system, [19] improves the internal force control performance by deducing the desired position; [20] implements the proposed method on the two manipulator systems to carry out hold-and-move task also in the teleoperation field and the authors separately handle the orientation, position, and force regulation problem. A symmetric strategy is developed in [21] for redundant dualarm robots to regulate both arm's pose and force coordination. In [22], a hybrid force/position controller is designed based on a fuzzy observer and a two-arm robot is applied to demonstrate the effectiveness in simulation. Researchers have done a great deal of work on achieving either better force control or the minimum position/orientation tracking errors. However, when they are in pursuit of a nice control performance on one side (e.g., force), they may sacrifice the performance on the other side (e.g., position/orientation).

Sometimes, we need a compromise between the behaviours of the force and the position; for example, when two robots are commanded to manipulate a deformable object from one place to another, they are informed to hold the object either with a desired internal force or along a predesigned trajectory (EE poses). However, few works take both of them into consideration in bimanual robot manipulation but just take one of the many items into account; for example, [23] focuses on only the position item. Hence, in this paper, we bend our effort for finding a suitable method to determine the optimal compromise when two robots cooperate in holding an object. Thanks are due to the linear-quadratic approaches presented in [24, 25], which, in a geometric way, explain the optimal intersection point of the force and the position in impedance model-based control. It gives us the inspiration to construct a similar cost function to estimate the control performance containing the force and the position tracking in the bimanual manipulation task. Methods to obtain the optimal solution to such kind of cost function in impedance model-based linear/ nonlinear system can be found in the literature; for example, [26] seeks help from iterative adaptive dynamic programming algorithm, and $[27,28]$ solve it by constructing the Hamilton-Jacobi-Bellman equation (HJB) while a neural network-based computational method is provided in [29] to handle the algebraic Riccati equation (ARE) that usually accompanies the HJB. The techniques in [29] make the solution to the HJB accessible, which is confirmed by researchers who apply the method into robot-environment interaction [30]. Inspired by these papers, in this paper, we construct a suitable performance measure to fit the bimanual robots' collaboration situation in order to gain an optimal regulation for force and position.

To facilitate the proposed method, we still need two auxiliary techniques to transfer the Cartesian tasks into robot joint space control problems. First, environmental forces surely affect the tracking performance of a robot; therefore, before we compensate the contact force acting on each $\mathrm{EE}$, we need to measure how heavy the contact force is at the current time. In this respect, [31] introduces several ideas that we can turn to; for example, the technique regarding generalized momentum [32] can be an appropriate choice and is adopted by many researchers to design force observer without installing sensors on EE [33-36]. Second, a specific coordinate in the task space is the target pose we need to command our EE to move to, but we can only indirectly control the joint actuator, not directly the EE. Hence, position mapping from the task space to the joint space should be calculated. In this regard, the closed-loop inverse kinematics (CLIK), which has been studied for decades [37-40], is a sophisticated algorithm to obtain joint positions in real time and becomes our option in this paper. In the latter sections, we are going to introduce these two supplementary approaches to make our proposed bimanual robotic system complete.

The contributions of this paper lie in the following:

(i) This paper constructs a relatively complete bimanual robot simulation system, which contains two 3-degree-of-freedom (DOF) robots (different from those simple 2-DOF robots employed in many papers) and provides basic and practical techniques to make this system function well when it carries out tasks. These techniques include inverse kinematics algorithm CLIK and force observer that plays the role of a force sensor installed on the EE. Such a basic platform, with multiple robots and basic control algorithms, provides possibilities for the researcher to conduct simulation within a larger range of application scenarios.

(ii) We take both the force and the position regulation into consideration in the scenario that bimanual robots cooperate to hold an object. Despite the fact that we might be not able to minimize both tracking errors of the two factors (force and position), we make the sum of the two closed to the minimum values by modelling the object as an impedance model and constructing proper cost function to suit the solving procedure. And to work out the optimal solution, a model-free computational algorithm is introduced.

The structure of the paper is organized as follow: Section 2 (Methods) develops the proposed method, including the restatement on the purpose of this paper, some preliminary knowledge, and the introduction of the force observer and the CLIK; right after Methods comes Section 3 (Simulations and Analysis) that involves the simulation configuration, procedures, results, and analysis; the final section (Conclusions) summarizes the whole paper.

\section{Methods}

In this section, we are going to (1) explain the optimal target (namely, the significance) of minimizing the sum of the tracking errors of both the force and the position, (2) 
develop the adaptive optimal method on regulation on force and position of bimanual robots in holding an object, and (3) present the preliminary techniques (force observer and CLIK) that contribute to the bimanual manipulating system. A brief introduction of the whole control system of the bimanual robots is depicted in Figure 1, which will be explained gradually in the following subsections.

2.1. Geometrical Interpretation on Minimizing the Sum of the Errors. When robots interact with physical objects in the form of an impedance model, it is usually commanded to "touch" them with its EE moving to a certain position or approaching the object's centre point until it senses a desired counterforce. In these cases, a single error of either force or position is considered and can be properly minimized. However, in many other cases, we need the tracking of both the force and the position to reach a relatively good performance rather than just one of them. And the following are the explanation of what the relatively "good" point (the optimal target) is. be

Let us consider a deformable object's physical model to

$$
f=g(\ddot{x}, \dot{x}, x),
$$

where $f$ denotes the subjected force, $x, \dot{x}$, and $\ddot{x}$ represent the deformation, deforming velocity, and deforming acceleration of the object, respectively, and $g(\cdot)$ is the mapping with regard to the object's impedance model.

In implementation, we desire that the object subjects to a certain force $f_{d}$ with a certain deformation state $x_{d}$, but we do not know the exact parameters in $g(\cdot)$. Hence, in most cases, $f_{d} \neq g\left(\ddot{x}, \dot{x}, x_{d}\right)$. Therefore, referring to $[24,25]$, we can obtain an optimal point $\left(f_{\text {opt }}, x_{\text {opt }}\right)$ that is the nearest to the desired set $\left(f_{d}, x_{d}\right)$ and satisfies the condition $f_{\text {opt }}=g\left(\ddot{x}, \dot{x}, x_{\text {opt }}\right)$. It can be explained geometrically via Figure 2 [25].

Note 1. If we take $\dot{x}$ or $\ddot{x}$ into consideration, it will turn out to be a three- or four-dimensional optimization problem.

Only in such an optimal point $\left(f_{\text {opt }}, x_{\text {opt }}\right)$, can the sum of tracking errors of the force and the deformation be the minimum; namely,

$$
\left(f_{\mathrm{opt}}-f_{d}\right)^{2}+\left(x_{\mathrm{opt}}-x_{d}\right)^{2}=\min \left\{\left(f-f_{d}\right)^{2}+\left(x-x_{d}\right)^{2}\right\} .
$$

If we add weights $W_{f}$ and $W_{x}$ to the tracking errors in equation (2), it turns out to be

$$
\begin{aligned}
& W_{f}\left(f_{\mathrm{opt}}-f_{d}\right)^{2}+W_{x}\left(x_{\mathrm{opt}}-x_{d}\right)^{2} \\
& \quad=\min \left\{W_{f}\left(f-f_{d}\right)^{2}+W_{x}\left(x-x_{d}\right)^{2}\right\} .
\end{aligned}
$$

In the case of bimanual robots holding an object, $f$ becomes the object's internal force and $x$ equals the distance between the EEs of the two robotic arms. We can adjust weights $W_{f}$ and $W_{x}$ to achieve different tracking performance. Particularly, if $W_{f}=0$ and $W_{f} \neq 0$, that is, pure

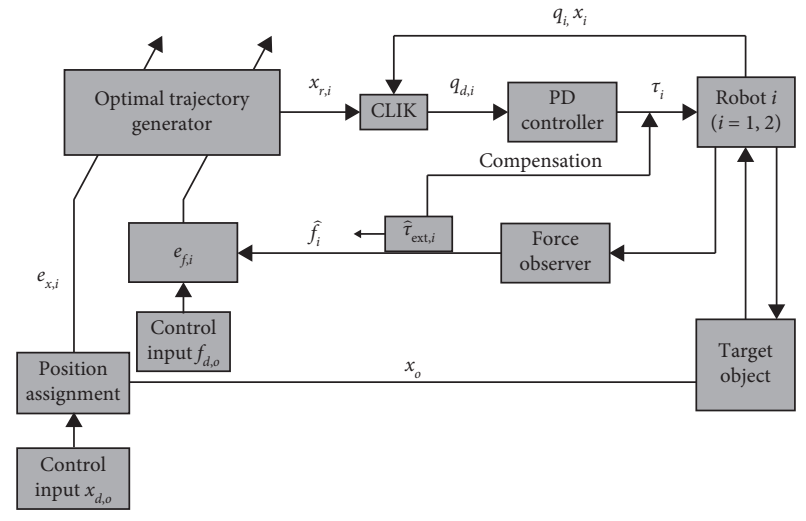

FIgURE 1: The whole system structure of the bimanual robot manipulation.

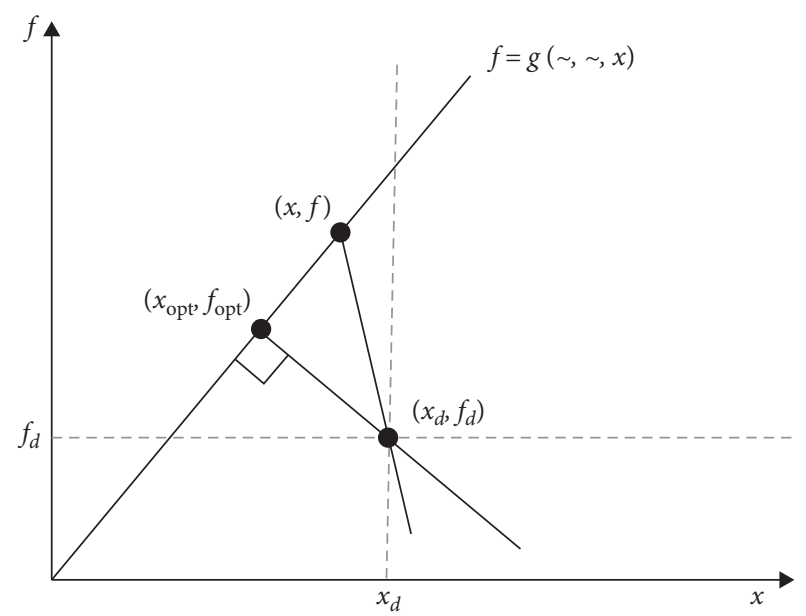

Figure 2: A 2D graph to explain the geometrical optimal point of the force and the deformation, which locates at the intersection between the line $f=g(\sim, \sim, x)$ and the line perpendicular to it and passing through $\left(f_{d}, x_{d}\right)$.

position control, while $W_{f} \neq 0$ with $W_{x}=0$ represents pure force control.

Considering the 3D space of the task space, $f$ and $x$ denote generalize force (force and torque) and position (position and orientation) with 6 dimensions in each; the performance measure can be written in a quadratic form as

$$
\Gamma=e_{f}^{T} W_{f} e_{f}+e_{x}^{T} W_{x} e_{x},
$$

where $e_{f}=f-f_{d}$ and $e_{x}=x-x_{d}$ are tracking errors of force and position.

If we need to guarantee the tracking performance within a certain time interval $\left[t_{a}, t_{b}\right]$, equation (4) needs to be modified into equation (5):

$$
\Gamma=\int_{t_{a}}^{t_{b}}\left(e_{f}^{T} W_{f} e_{f}+e_{x}^{T} W_{x} e_{x}\right) \mathrm{d} t .
$$

By minimizing equation (5), we can obtain the optimal performance from $t_{a}$ to $t_{b}$. 
2.2. Adaptive Optimal Regulation on Force and Position. In object holding tasks, as we do not move the object, we ignore the acceleration information. Let us consider the object to be held as a damping-spring model whose expression is written as

$$
-f_{o}=C_{o} \dot{x}_{o}+K_{o} x_{o}
$$

which is a specific situation of equation (1) with $x_{o}$ and $\dot{x}_{o}$ denoting the object's deformation and its deforming velocity and $f_{o}$ standing for its internal force.

In such case with the object being a damping-spring model, considering the preliminary knowledge in the previous subsection, with reference to $[30,34]$, the cost function is set to be

$$
\begin{aligned}
\Gamma_{m}= & \int_{0}^{t_{f}}\left[\left(x_{o}-x_{d, o}\right)^{T} W_{x}\left(x_{o}-x_{d, o}\right)\right. \\
& \left.+\left(\hat{f}_{o}-f_{d, o}\right)^{T} W_{f}\left(\hat{f}_{o}-f_{d, o}\right)\right] \mathrm{d} t,
\end{aligned}
$$

where $t_{f}$ stands for the system running final time; $x_{d, o}, \widehat{f}_{o}$, and $f_{d, o}$ are the user-desired deformation, the estimated subjected force (the real internal force is usually inaccessible and the estimated value is obtained through force observer), and the user-desired object subjected force, respectively; $W_{x} \geq 0$ and $W_{f} \geq 0$ denote the weights for trajectory tracking error and force tracking error, respectively. $W_{x}$ and $W_{f}$ can be defined according to the tracking demand; for example, if we require precision in force tracking, $W_{x}$ can be set to closed 0 while $W_{f} \neq 0$, and likewise, the situation with $W_{x} \neq 0$ and $W_{f}$ closed to 0 makes the position tracking more accurate.

The absolute value of subjected force on EE $f_{\text {ext, } i}$ (the subscript $i=1,2$ is used to distinguish between robot 1 and robot 2) equals the internal force of object $f_{o}$ in a relatively static condition and the estimation of the subjected force on $\mathrm{EE} \widehat{f}_{\text {ext }, i}$ usually converges to the real value. In this paper and in the application scenarios, we have

$$
f_{o}=\left|f_{\text {ext }, i}\right|=\left|\hat{f}_{\text {ext }, i}\right|=\hat{f}_{o}
$$

In this paper, we want the object internal force to be minimized when it is held by two robots, namely, using a minimum force to hold an object; therefore, we set the desired internal force:

$$
f_{d, o}=0 .
$$

Then, equation (7) in this paper can be rewritten as

$$
\Gamma_{m}=\int_{0}^{t_{f}}\left[\left(x_{o}-x_{d, o}\right)^{T} W_{x}\left(x_{o}-x_{d, o}\right)+\left(\hat{f}_{\text {ext, }, i}\right)^{T} W_{f}\left(\hat{f}_{\text {ext }, i}\right)\right] \mathrm{d} t .
$$

In order to employ the optimal control [29] in trajectory tracking problem, we define a linear system to determine the $x_{d, o}$ as

$$
\begin{aligned}
\dot{\eta}_{o} & =U \eta_{o}, \\
x_{d, o} & =V \eta_{o},
\end{aligned}
$$

where $\eta_{0}$ is treated as a system state; $U$ and $V$ are two matrices to be determined. Then equation (7) can be rewritten as

$$
\begin{aligned}
\Gamma_{m} & =\int_{0}^{t_{f}}\left\{\left[\begin{array}{ll}
x_{o}^{T} & x_{d, o}^{T}
\end{array}\right]\left[\begin{array}{cc}
W_{x} & -W_{x} V \\
-V^{T} W_{x} & V^{T} W_{x} V
\end{array}\right]\left[\begin{array}{c}
x_{o} \\
x_{d, o}
\end{array}\right]+e_{f}^{T} W_{f} e_{f}\right\} \mathrm{d} t \\
& =\int_{0}^{t_{f}}\left\{\xi^{T} Q \xi+e_{f}^{T} \operatorname{Re}_{f}\right\} \mathrm{d} t,
\end{aligned}
$$

where $\quad \xi=\left[\begin{array}{ll}x_{o}^{T} & \eta_{o}^{T}\end{array}\right]^{T}, \quad e_{f}=\widehat{f}_{\text {ext }, i}-f_{d, o}=\widehat{f}_{\text {ext }, i}$, $Q=\left[\begin{array}{cc}W_{x} & -W_{x} V \\ -V^{T} W_{x} & V^{T} W_{x} V\end{array}\right]$, and $R=W_{f}$. Let us view $\xi$ as a state in another system, modified from equation (6), whose state-space form is

$$
\dot{\xi}=A \xi+B e_{f},
$$

where $A=\left[\begin{array}{cc}-C_{o}^{-1} K_{o} & 0 \\ 0 & U\end{array}\right]$ and $B=\left[\begin{array}{c}-C_{o}^{-1} \\ 0\end{array}\right]$.

In this system, $e_{f}$ is treated as the system input. Through linear optimal control theory [41] and the proven model-free adaptive dynamic programming (ADP) algorithm in [29], an optimal control law

$$
e_{f}=-K_{\mathrm{opt}} \xi
$$

can be acquired such that the performance index (equation (12)) is minimized without knowing $A$ or $B$. The key point is to obtain the feedback gain matrix $K\left(\longrightarrow K_{\text {opt }}\right)$, which can be computed via the flowing steps (refined and depicted in Figure 3).

Step 1. Take $e_{f}=-K_{0} \xi+\nu$ as the input on the time interval $\left[t_{0}, t_{l}\right]$, in which $K_{0}$ is stabilizing and $v$ is the exploration noise to meet the persistent excitation (PE) condition. Compute $\delta_{\xi, \xi}, I_{\xi, \xi}$, and $I_{\xi, e_{f}}$ through

$$
\begin{aligned}
\delta_{\xi, \xi} & =\left[\bar{\xi}\left(t_{1}\right)-\bar{\xi}\left(t_{0}\right), \bar{\xi}\left(t_{2}\right)-\bar{\xi}\left(t_{1}\right), \ldots, \bar{\xi}\left(t_{l}\right)-\bar{\xi}\left(t_{l-1}\right)\right]^{T}, \\
I_{\xi, \xi} & =\left[\int_{t_{0}}^{t_{1}} \xi \otimes \xi \mathrm{d} t, \int_{t_{1}}^{t_{2}} \xi \otimes \xi \mathrm{d} t, \ldots, \int_{t_{l-1}}^{t_{l}} \xi \otimes \xi \mathrm{d} t\right]^{T}, \\
I_{\xi, e_{f}} & =\left[\int_{t_{0}}^{t_{1}} \xi \otimes e_{f} \mathrm{~d} t, \int_{t_{1}}^{t_{2}} \xi \otimes e_{f} \mathrm{~d} t, \ldots, \int_{t_{l-1}}^{t_{l}} \xi \otimes e_{f} \mathrm{~d} t\right]^{T},
\end{aligned}
$$

with “ $\otimes$ ” denotes the Kronecker product and

$$
\bar{\xi}=\left[\xi_{1}^{2}, \xi_{1} \xi_{2}, \ldots, \xi_{1} \xi_{p}, \xi_{2}^{2}, \xi_{2} \xi_{3}, \ldots, \xi_{2} \xi_{p}, \ldots, \xi_{p-1}^{2}, \xi_{p-1} \xi_{p}, \xi_{p}^{2}\right]^{T},
$$

until the following condition is satisfied:

$$
\operatorname{rank}\left(\left[I_{\xi, \xi}, I_{\xi, e_{f}}\right]\right)=\frac{p(p+1)}{2}+p r
$$

where $p$ is the number of elements in $\xi$ and $r$ is the number of elements in $e_{f}$.

Step 2. Solve $P_{k}$ and $K_{k+1}$ according to 


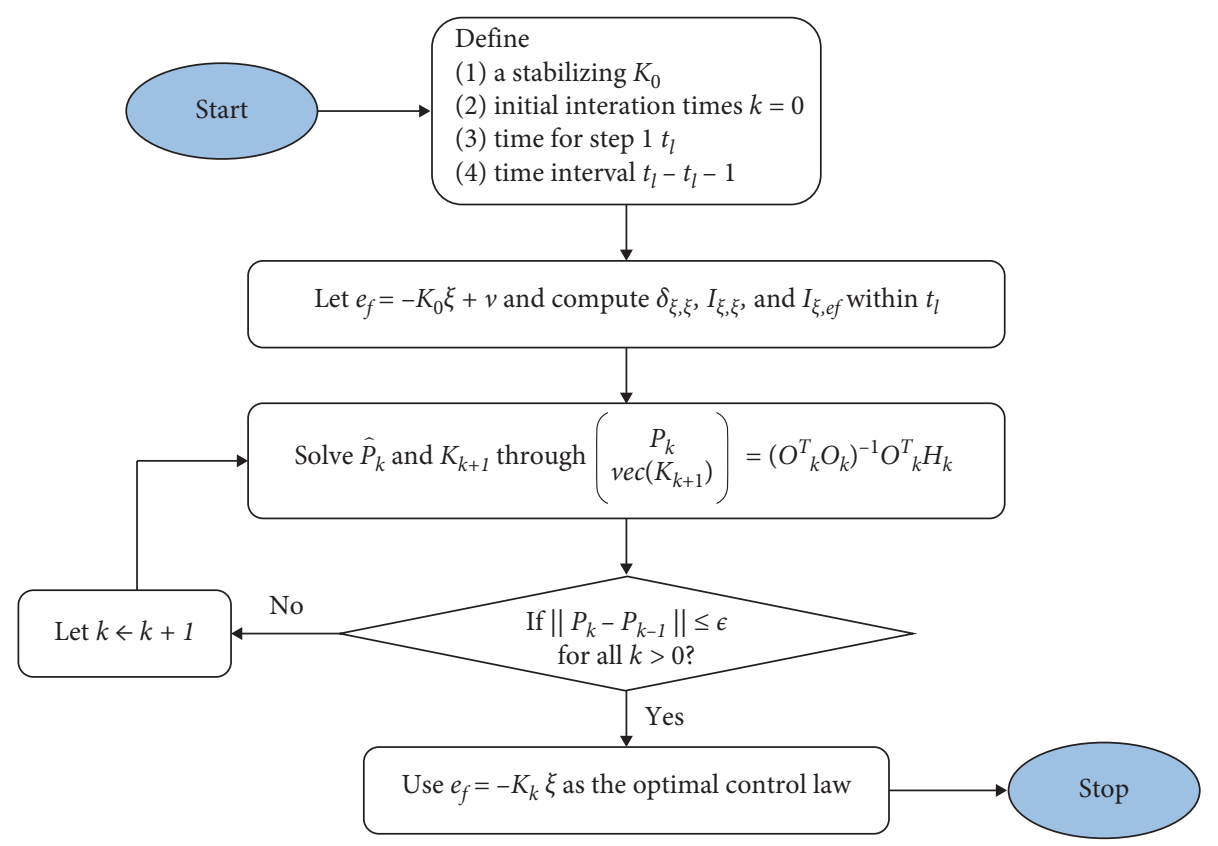

FIgURE 3: The flowchart of the procedures for obtaining the approximate optimal control law.

$$
\left[\begin{array}{c}
\widehat{P}_{k} \\
\operatorname{vec}\left(K_{k+1}\right)
\end{array}\right]=\left(\Theta_{k}^{T} \Theta_{k}\right)^{-1} \Theta_{k}^{T} \Xi_{k}
$$

where "vec" is the operator that "stretches" a matrix to a vector; that is, if $X=\left[c_{1}, c_{2}, c_{3}\right]$ with $c_{1}, c_{2}$, and $c_{3}$ are column vectors, then $\operatorname{vec}(X)=\left[c_{1}^{T}, c_{2}^{T}, c_{3}^{T}\right]^{T}$, and

$$
\begin{aligned}
\widehat{P}_{k}= & {\left[P_{1,1}, 2 P_{1,2}, \ldots, 2 P_{1, p}, P_{2,2}, 2 P_{2,3}, \ldots, 2 P_{2, p},\right.} \\
& \left.\ldots, P_{p-1, p-1}, 2 P_{p-1, p}, P_{p, p}\right]^{T}, \\
\Theta_{k}= & {\left[\delta_{\xi, \xi},-2 I_{\xi, \xi}\left(I_{p} \otimes K_{k}^{T} R\right)-2 I_{\xi, e_{f}}\left(I_{p} \otimes R\right)\right], } \\
\Xi_{k}= & -I_{\xi, \xi} \operatorname{vec}\left(Q_{k}\right),
\end{aligned}
$$

where $I_{p}$ is a unit matrix with $p$ dimensions, $P_{i, j}(i, j=$ $1,2,3, \ldots, p)$ are the elements of $P_{k}$, and

$$
Q_{k}=Q+K_{k}^{T} R K_{k} \text {. }
$$

Note 2. The solution of $P_{*}$ is the key point to the wellknown algebraic Riccati equation (ARE) before deriving the optimal feedback gain matrix $K_{\text {opt }}[29]$.

Step 3. Let $k \longleftarrow k+1$ and repeat Step 2 until $\left\|P_{k}-P_{k-1}\right\| \leq \varepsilon$, where $\varepsilon>0$ is a predefined constant.

Step 4. Use equation (21) as the approximate optimal control law.

$$
e_{f}=-K_{k} \xi
$$

Remark 1. It is noticed that the above procedure does not rely on $A$ or $B$ in equation (13), which shows one of its favourable properties that the optimal solution is applicable to that system with unknown dynamics parameters, for example, an object with unknown mass, damping, or spring parameters. In many application scenarios, these parameters are usually inaccessible.

2.3. Generalized-Momentum-Based Force Observer. As mentioned in equation (7), we need to observe the robot's subjected force to replace the object's internal force. Thus, in this subsection, the target is to construct a force observer to "sense" the force act on the EE without installing sensor hardware on it.

The two-robots dynamics model in the bimanual system can be expressed as

$$
\left\{\begin{array}{l}
M_{1}\left(q_{1}\right) \ddot{q}_{1}+C_{1}\left(q_{1}, \dot{q}_{1}\right) \dot{q}_{1}+G_{1}\left(q_{1}\right)=\tau_{1}+\tau_{\text {ext, },} \\
M_{2}\left(q_{2}\right) \ddot{q}_{2}+C_{2}\left(q_{2}, \dot{q}_{2}\right) \dot{q}_{2}+G_{2}\left(q_{2}\right)=\tau_{2}+\tau_{\text {ext, } 2}
\end{array}\right.
$$

where $q_{i} \in R^{n_{i}}$ stands for the joint position of robot $i$ ( $i=$ $1,2) \quad\left(n_{i}\right.$ is the joint number of robot $\left.i\right) ; M_{i}\left(q_{i}\right) \in R^{n_{i} \times n_{i}}$ denotes the inertia matrix of the robot $i$; $C_{i}\left(q_{i}, \dot{q}_{i}\right) \in R^{n_{i} \times n_{i}}$ is the centripetal and Coriolis coupling matrix; $G_{i}\left(q_{i}\right) \in R^{n_{i}}$ represents the gravitational force; $\tau_{i} \in R^{n_{i}}$ is the control input torque, and $\tau_{\text {ext,i }} \in R^{n_{i}}$ is the torque act on joints caused by the subjected force $f_{\text {ext }, i}$ on the EE.

Property 1. $M_{i}\left(q_{i}\right)$ is a symmetric and positive definite matrix [42].

Property 2. The result of $\dot{M}_{i}\left(q_{i}\right)-2 C_{i}\left(q_{i}, \dot{q}_{i}\right)$ is a skewsymmetric matrix [42].

As it is known [43], the relationship between $\tau_{\mathrm{ext}, i}$ and $f_{\text {ext, } i}$ is

$$
f_{\mathrm{ext}, i}=J_{i} \tau_{\mathrm{ext}, i}
$$


where $J_{i}$ is the Jacobian matrix of robot $i$. Hence, we can estimate $f_{\text {ext,i }}$ indirectly via observing $\tau_{\text {ext,i }}$. Referring to the concept of generalized momentum [32] and its applications [33-36], we define $p_{i}$ as the generalized momentum with

$$
p_{i}=M_{i} \dot{q}_{i}
$$

Then, we have

$$
\begin{aligned}
\dot{p}_{i} & =M_{i} \ddot{q}_{i}+\dot{M}_{i} \dot{q}_{i} \\
& =\dot{M}_{i} \dot{q}_{i}+\tau_{i}+\tau_{\text {ext }, i}-C_{i} \dot{q}_{i}-G_{i},
\end{aligned}
$$

and with properties in [42], the derivative of inertia matrix $M$ with respect to time is

$$
\dot{M}_{i}=C_{i}+C_{i}^{T} .
$$

Substituting equation (26) into equation (25), we obtain

$$
\begin{aligned}
\dot{p}_{i} & =C_{i}^{T} \dot{q}_{i}+\tau_{i}-G_{i}+\tau_{\mathrm{ext}, i} \\
& =u_{i}+\tau_{\mathrm{ext}, i},
\end{aligned}
$$

where we define $u_{i}=C_{i}^{T} \dot{q}_{i}+\tau_{i}-G_{i}$.

According to $[32,36]$, the observer for the generalized momentum $p_{i}$ can be written as

$$
\dot{\hat{p}}_{i}=u_{i}+L_{i}\left(p_{i}-\widehat{p}_{i}\right)
$$

where

$$
\widehat{\tau}_{\text {ext, } i}=L_{i}\left(p_{i}-\widehat{p}_{i}\right)
$$

is the estimation of $\tau_{\text {ext, } i}$. Next, we obtain the estimated value of the subjected force $f_{\text {ext, } i}$ with the help of equation (23). That is,

$$
\widehat{f}_{\text {ext }, i}=J_{i} \widehat{\tau}_{\text {ext }, i},
$$

which will be used to compare with the desired contact force $f_{d, i}\left(=f_{d, o}=0\right)$.

2.4. EE Pose Assignment and CLIK Algorithm. The basic problem of the bimanual robot system is to drive the robot EEs to first touch and then hold the object. In this aspect, we should have the desired trajectory and the way to convert the trajectory into desired Cartesian space to joint angles.

So far, the optimal control input $e_{f}^{\text {opt }}$ is obtained according to equation (21), then, the optimal deformation is derived

$$
x_{o}^{\mathrm{opt}}=-\frac{1}{K_{1}}\left(e_{f}^{\mathrm{opt}}+K_{2} \eta_{o}\right)
$$

where $\left[K_{1} K_{2}\right]=K_{k}$.

Considering the object being held by two manipulators, we have to transform the optimal deformation $x_{o}^{\text {opt }}$ of the object into certain reference positions $x_{r, i}$ for each EE. To this end, we apply a simple symmetric way (a more complicated way is introduced in [21]) for robots to hold the object. As depicted in Figure 4, the reference robots' EE position $x_{r, i}$ is obtained

$$
x_{r, i}=x_{O}+T_{i}
$$

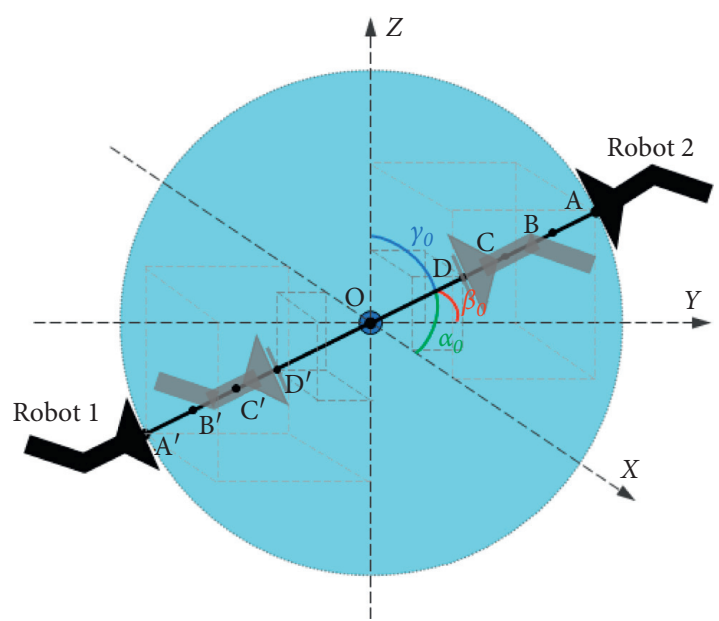

$$
\begin{aligned}
& \mathrm{AA}^{\prime} \text { : two EEs just touch the object. } \\
& \mathrm{BB}^{\prime} \text { : a deformation (distance) for a desired force. } \\
& \mathrm{CC}^{\prime} \text { : an optimal deformation } x_{o} \text { opt. } \\
& \mathrm{DD}^{\prime} \text { : a desired deformation (distance). } \\
& \mathrm{O} \text { : the target holding centre. }
\end{aligned}
$$

FIgURE 4: A symmetric way to hold the object by two robots, where two EEs approach the object centre symmetrically.

where $x_{O}=\left[\begin{array}{c}P_{O} \\ O_{O}\end{array}\right]$ (different from object deformation $\left.x_{o}\right)$ is the target holding centre within the object with $P_{O}$ and $O_{O}$ denoting the object's position and orientation, respectively, and $T_{i}=\left[\begin{array}{c}T_{P_{i}} \\ T_{O_{i}}\end{array}\right]$ is the transformation from the centre to the EE with $T_{P_{i}}$ and $T_{O_{i}}$ being the position and the orientation transformation. To be specific,

$$
\begin{aligned}
& T_{P_{i}}= \pm \frac{1}{2} x_{o}^{\mathrm{opt}}\left[\begin{array}{c}
\cos \alpha_{o} \\
\cos \beta_{o} \\
\cos \gamma_{o}
\end{array}\right], \\
& T_{O_{i}}= \begin{cases}0_{3 \times 1}, & i=1, \\
-\pi I_{3 \times 1}, & i=2,\end{cases}
\end{aligned}
$$

where $\alpha_{o}, \beta_{o}$, and $\gamma_{o}$, as shown in Figure 4, represent the desired orientation of the object and $i=1$ and 2 represents the robot $i$ on the left $(i=1)$ and the right $(i=2)$ of the object.

Finally, the Cartesian space coordinate $x_{r, i}$ can be reached through CLIK [37-40]. From Figure 1, we can see that we need to derive desired joint position $q_{d, i}$ based on the information of the robot's current state $\left(q_{i}\right.$, and $\left.x_{i}\right)$ and the target EE position $x_{r, i}$.

In CLIK, the inverse kinematics problem of a class of serial-link manipulators is treated as a dynamical problem. As we usually use serial-link robots in the bimanual manipulator system, the computation from the joint space velocity to the task space velocity is described as

$$
\dot{q}_{d, i}=J_{i}^{\dagger}\left(\dot{x}_{r, i}+k_{E} e_{x_{r}, i}\right)
$$


where $e_{x_{r, i}}=x_{i}-x_{r, i}$ is the EE tracking error.

Then, the target joint position, which is employed as the input of the PD controller in Figure 1, is derived by

$$
q_{d, i}=\int_{0}^{t_{c}} J_{i}^{\dagger}\left(\dot{x}_{r, i}+k_{E} e_{x_{r}, i}\right) \mathrm{d} t
$$

where $t_{c}$ represents the current time.

\section{Simulations and Analysis}

In this section, we conduct two groups of simulations on MATLAB platform, in which two 3-DOF robots are employed to hold an object. The first group aims to demonstrate the importance of the force observer and the CLIK. The second group mainly shows the significance of the synchronously optimal regulation on force and position in bimanual robots holding tasks. Some basic information on the bimanual robot system is listed in Table 1.

3.1. Simulation Group 1. In the first group of simulation, two cases are conducted. Case 1 is a demo, commanding two robot EEs to collaborate to approach an object before holding it. Case 2 is a comparison between the situations with and without using force observer.

Case 1. In this case, we suppose that there exists an object $\left(C_{o}=0.01, K_{o}=1\right)$ that (randomly) locates within the intersection of the two robots' workspace, shown in Figure 5. From $0 \mathrm{~s}$ to $2 \mathrm{~s}$, we generate two paths for both robots, and they move their EEs from the initial positions to the holding target positions through the derived desired joint position $q_{d, i}$ obtained via CLIK. From $2 \mathrm{~s}$ to $6.5 \mathrm{~s}$, the two robots are commanded to hold the object according to the desired object desired deformation trajectory $x_{o}=0.2\left(1-e^{-(t-2)}\right)$, where $U=-1$ and $V=0.2$. Finally, from $6.5 \mathrm{~s}$ to $10 \mathrm{~s}$, based on the aforementioned pose assignment method and according to the desired object poses, new EE trajectories are generated. And as robots move along the two paths, the object also turns along the desired poses. Some sample frames are posed in Figure 5, and some changing variables as time goes by are depicted in Figure 6 .

Based on the results, it is not hard to tell that (1) the CLIK algorithm plays an important role in generating the $q_{d, i}$, enabling EEs to move precisely along the desired path so as to enable the object to deform and turn along the desired trajectory (Figures 6(e)-6(f)); (2) the force observer performs well in estimating the subjected forces acting on EEs such that the estimated object internal force is worked out (Figures 6(b) and 6(c)).

Case 2. In this case, we demonstrate the importance of the developed force observer by comparing the deformation tracking errors (namely, the tracking performance of EEs) in the conditions with and without using force observer. We place the object in the centre between the initial position of EEs, seen in Figure 7, and then command the two robots to hold the object for a while along the desired trajectory $x_{d, o}=0.2\left(1-e^{-t}\right)$. The object's deformation information
TABLe 1: Partial parameters of the bimanual robot system.

\begin{tabular}{lc}
\hline Items & Values \\
\hline Robot 1 link length & $l 11, l 12, l 13=0.5,0.3,0.1(\mathrm{~m})$ \\
Robot 2 link length & $l 21, l 22, l 23=0.5,0.3,0.1(\mathrm{~m})$ \\
Robot 1 link mass & $m 11, m 12, m 13=1.5,1.0,0.5(\mathrm{~kg})$ \\
Robot 2 link mass & $m 21, m 22, m 23=1.5,1.0,0.5(\mathrm{~kg})$ \\
Robot 1 initial joint & {$[(2 / 3) \pi,-(1 / 3) \pi,-(1 / 3) \pi]^{T}(\mathrm{rad})$} \\
position & {$[(1 / 3) \pi,(1 / 3) \pi,(1 / 3) \pi]^{T}(\mathrm{rad})$} \\
Robot 2 initial joint & {$[0,0](\mathrm{m})$} \\
position & {$[0.5,0](\mathrm{m})$} \\
Robot 1 base coordinate & $g=0$ \\
Robot 2 base coordinate & $10^{-4}(\mathrm{~s})$ \\
Gravitational acceleration & $K p=10^{2} \operatorname{diag}[40,35,28]$ \\
Sample time & $K d=\operatorname{diag}[10,1,0.01]$ \\
PD controller gains &
\end{tabular}

collected from the two conditions is illustrated in Figure 8. It is apparent that, under the circumstance without employing force observer, the subjected force is not sensed, and thus, the compensation for the joint torque is 0 resulting in that the PD controller is always not able to address the EE position steady-state tracking error. We can also see larger steady-state errors as the external force goes up when using the same holding path but different object impedance parameters $\left(K_{o}=1,5,10\right)$ of the object. We can conclude that, without force observer, the steady-state errors stay larger as the external force becomes heavier.

Therefore, we can tell that, in the task of bimanual robots holding an object, the compensation for the contact force is of great importance. In other words, the force observer acts like a sensor installed on the EE and senses the contact force before informing the system to make up for the joint control torques so as to enable EEs to precisely move along the desired paths. In order to demonstrate the effectiveness of the force observer in estimating the subjected external torques and forces, some other pieces of information corresponding to the simulation of Figure $8(\mathrm{~g})$ are displayed in Figure 9. Figures 9(a)-9(d) show how the estimated joint torques (derived from equation (29)) and the estimated external forces (calculated from equation (30)) are closed to the real joint torques and the real subjected external forces, respectively, and Figure 9(e) illustrates slight error between the object's internal force and the resultant force of $\widehat{f}_{\text {ext, }, 2, x}$ and $\widehat{f}_{\text {ext,2,y }}$ (namely, the estimated object's internal force).

3.2. Simulation Group 2. In the second group of simulation, we also have two cases to illustrate how the impedance model-based optimal regulation method works. Case 3 shows a comparison between the optimal solutions obtained via the paper-introduced algorithm without knowing model parameters ( $A$ and $B$ in equation (13)) and the solutions by solving algebraic Riccati equation (ARE) based on known $A$ and $B$. Case 4 demonstrates how effectively and adaptively the optimal method can regulate the force and position under various conditions. 


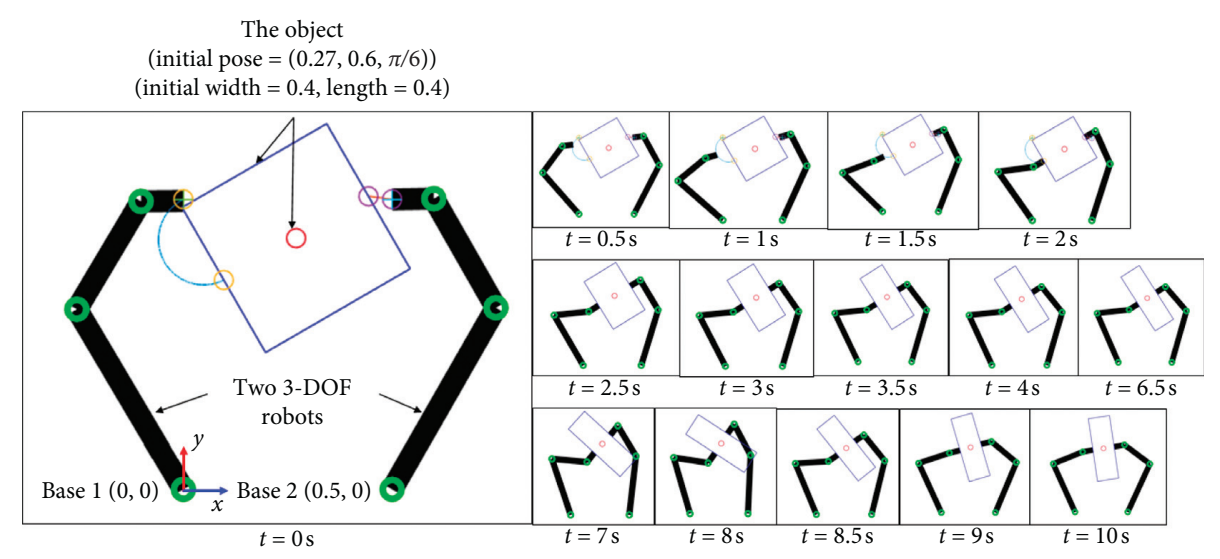

Figure 5: Some frames captured in the task described in Case 1.

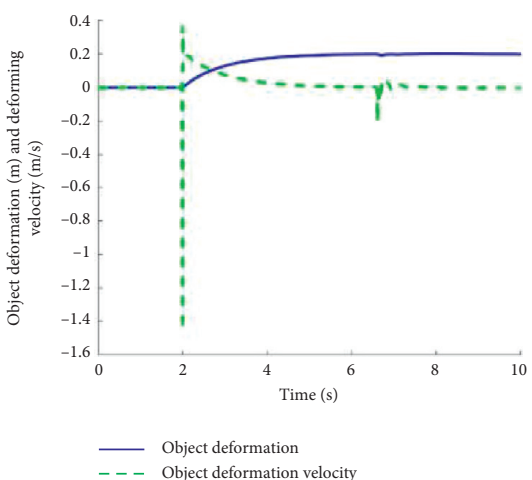

(a)

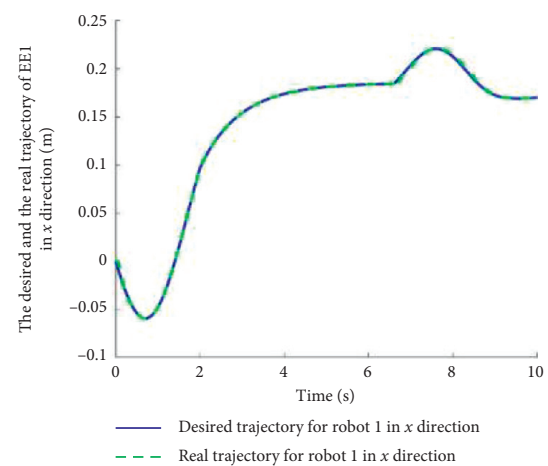

(d)

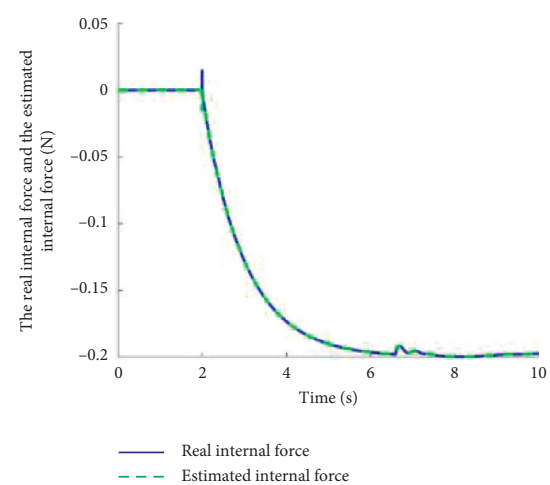

(b)

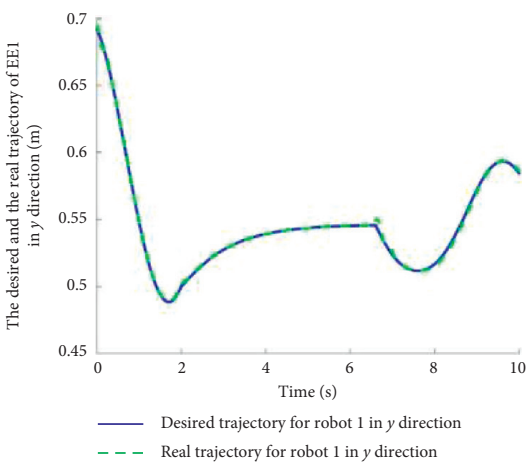

(e)

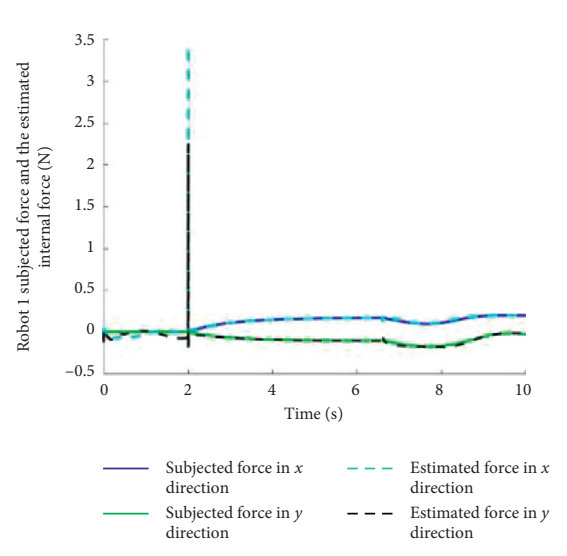

(c)

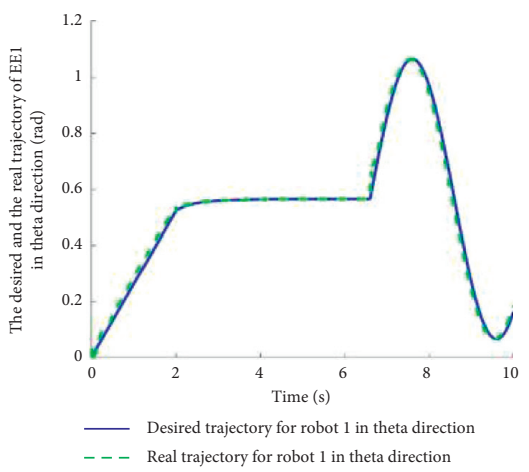

(f)

FIGURE 6: Some changing variables in Case 1, including object deforming information, internal force, estimated forces, and EE trajectories.

Case 3. In this case, the object impedance model is firstly set as $-f_{o}=0.01 \dot{x}_{o}+1 x_{o}$, and it is placed on the pose depicted in Figure 7; the other variables are $W_{x}=1, W_{f}=1, U=-1$, $V=0.2, \quad \varepsilon=0.03, \quad t_{l}-t_{l-1}=0.03, \quad K_{0}=[-1,1], \quad$ and $v=\sum_{i=1}^{8}(0.001 / i) \sin (i t)$. By exactly knowing these items, the optimal solution $K_{\mathrm{opt}}=[-0.4142,0.1404]$ is obtained through solving ARE, while the $K_{k}=[-0.4187,0.1324]$ is also gained without knowing the information of $A$ and $B$, through the aforementioned procedures (from Step 1 to Step $4)$. We can tell that $K_{k}$ is very closed to $K_{\text {opt }}$, and the control performance based on $K_{k}$ is also satisfactory according to the cost function results depicted in Figure 10(b), despite a gap between them, which does not affect the effectiveness of $K_{k}$ since the gap increment does not significantly tend to become larger after $2.5 \mathrm{~s}$. This gap is mainly accumulated within $0 \mathrm{~s}-2.5 \mathrm{~s}$, because (i) when executing Step 1 $(0 \mathrm{~s}-0.15 \mathrm{~s})$, a not very proper $K_{0}$ and the exploration noise $v$ are used; and, referring to Figure 10(a), (ii) to avoid a sudden big change between $x_{o}$ (obtained through $K_{0}$ in Step 1) and $x_{o}^{\mathrm{opt}}$ (obtained through $K_{k}$ via equation (31)), from $0.15 \mathrm{~s}$ on, 


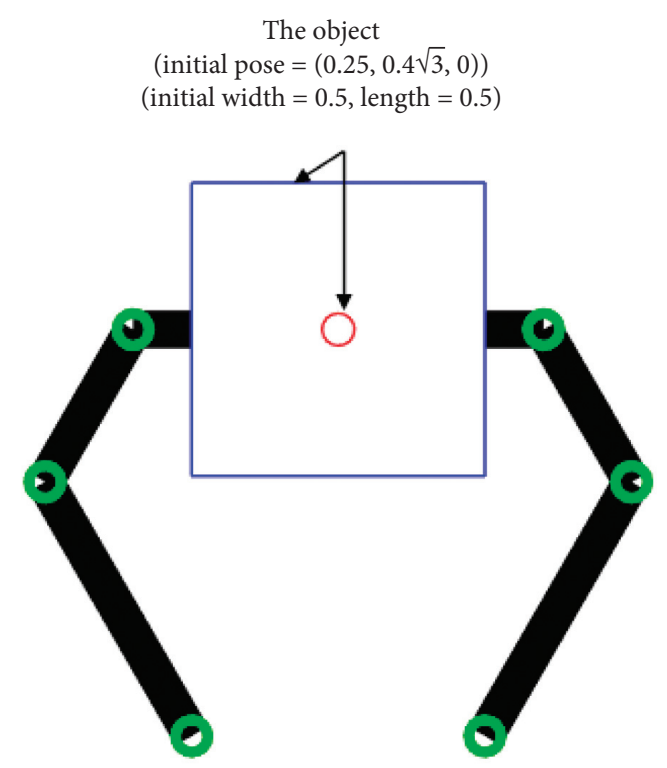

Figure 7: The initial state of the system in Cases 2-4.

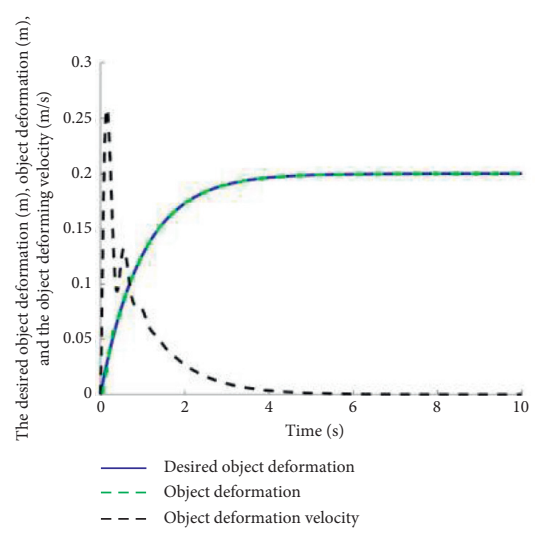

(a)

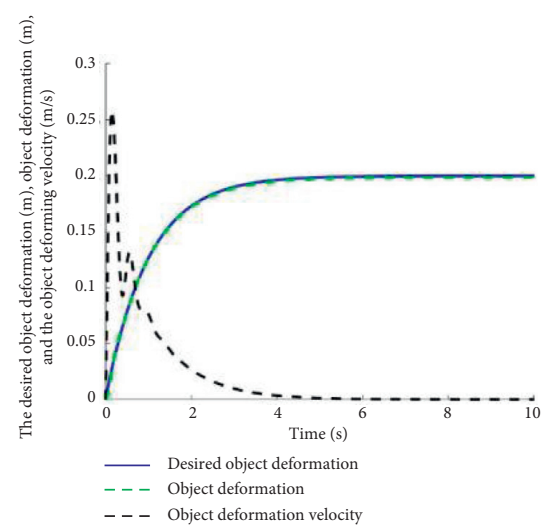

(d)

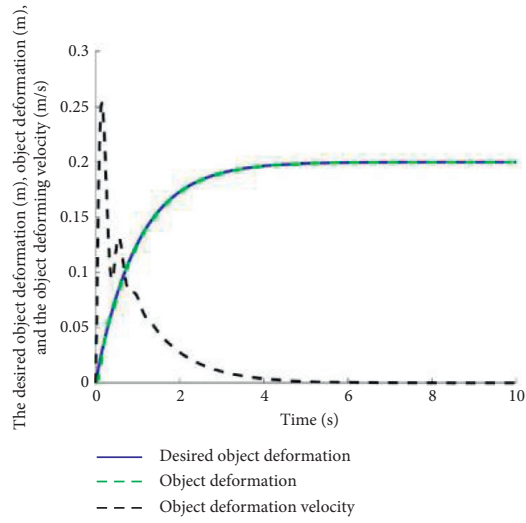

(b)

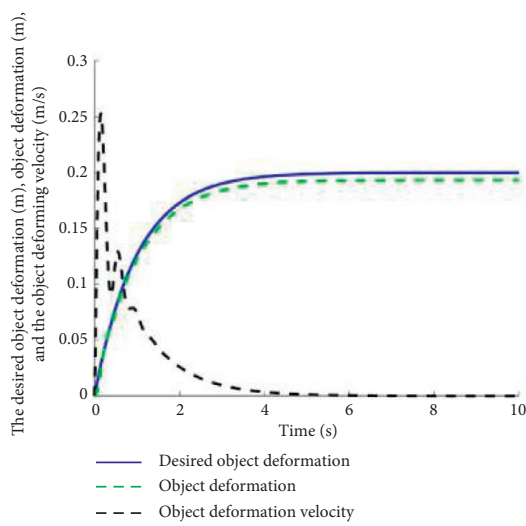

(e)

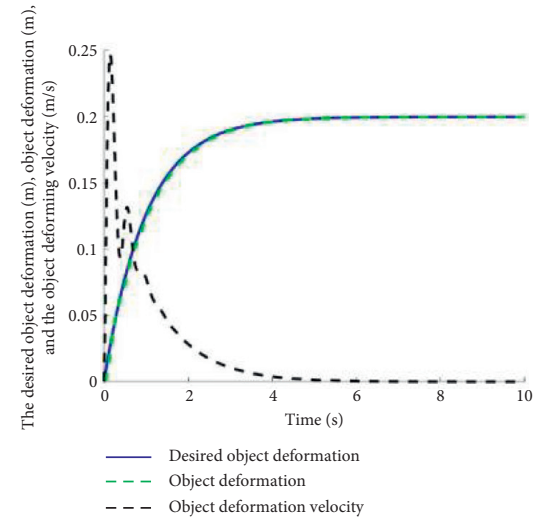

(c)

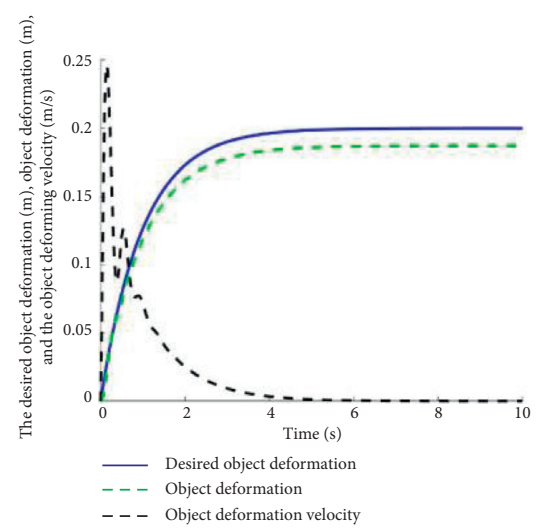

(f)

FIGURE 8: Comparison of deformation information of the object between the conditions with and without using force observer. (a) $K_{o}=1$ with force observer, (b) $K_{o}=5$ with force observer, (c) $K_{o}=10$ with force observer, (e) $K_{o}=1$ without force observer, (f) $K_{o}=5$ without force observer, and (g) $K_{o}=10$ without force observer. 


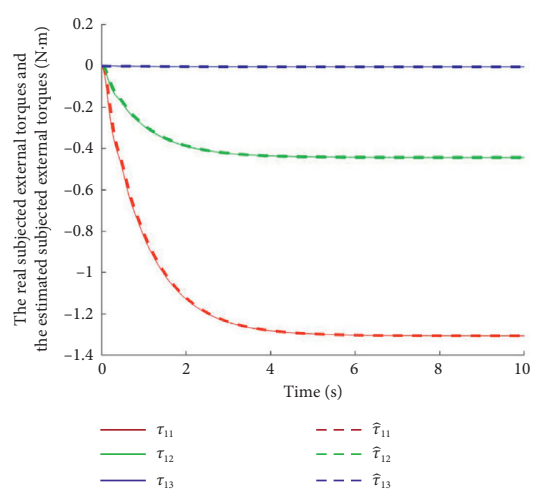

(a)
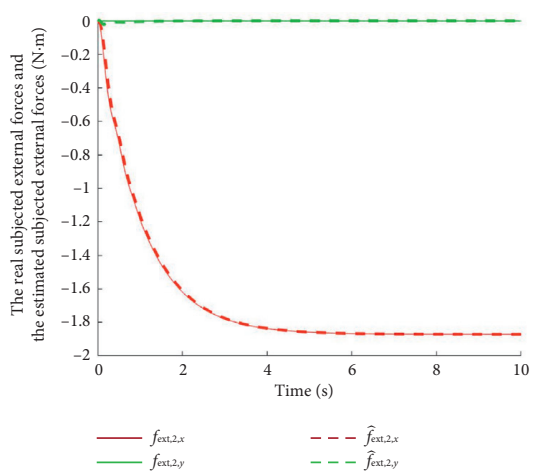

(d)

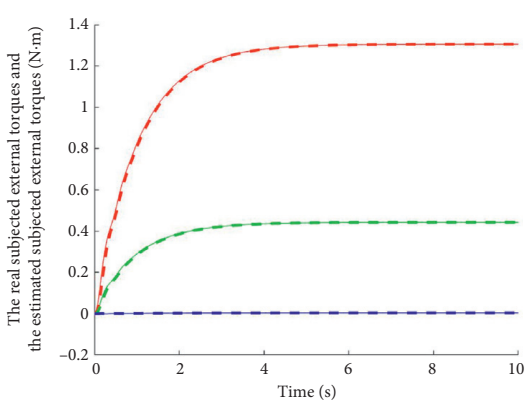

$---\hat{\tau}_{21}$
$---\hat{\tau}_{22}$
$----\hat{\tau}_{23}$

(b)

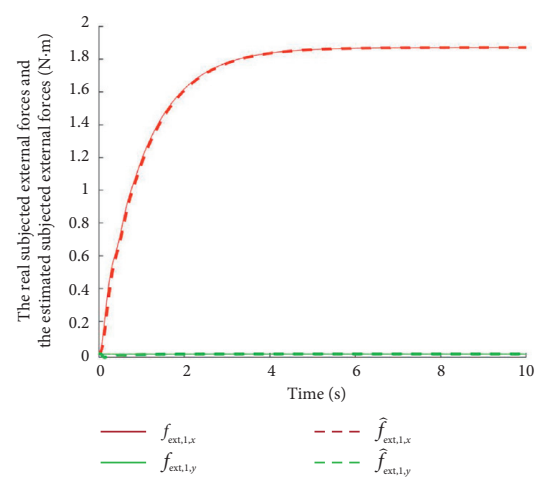

(c)

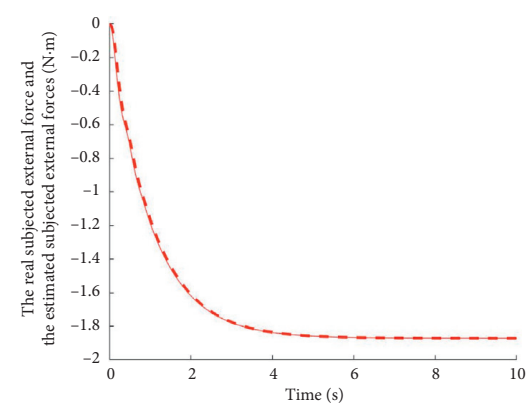

$-f_{o}$
$---f_{o},\left(\widehat{f}_{\text {ext }}\right)$

(e)

FIGURE 9: Some other system states corresponding to the simulation of Figure 8(g). (a) Subjected joint torques in robot 1. (b) Subjected joint torques in robot 2. (c) Subjected external force in EE 1. (d) Subjected external force in EE 2. (e) Object's internal force.

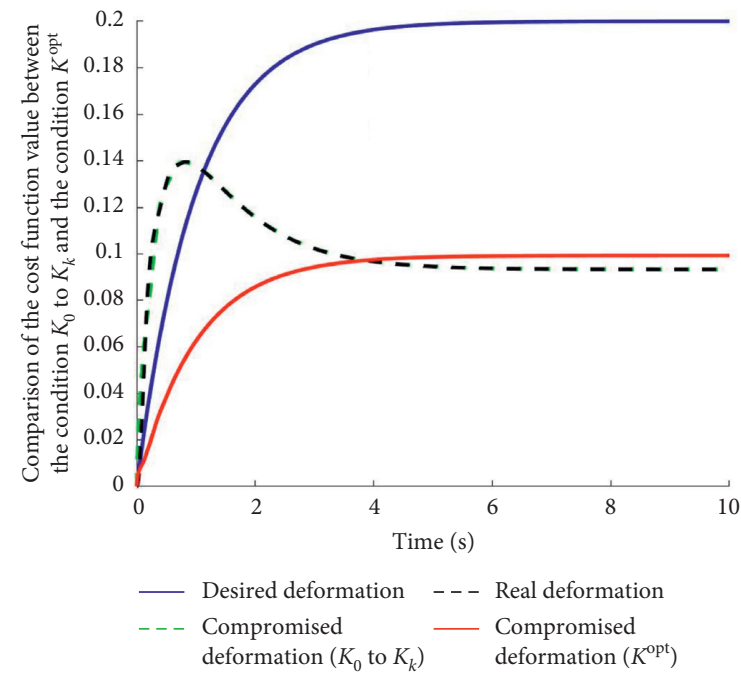

(a)

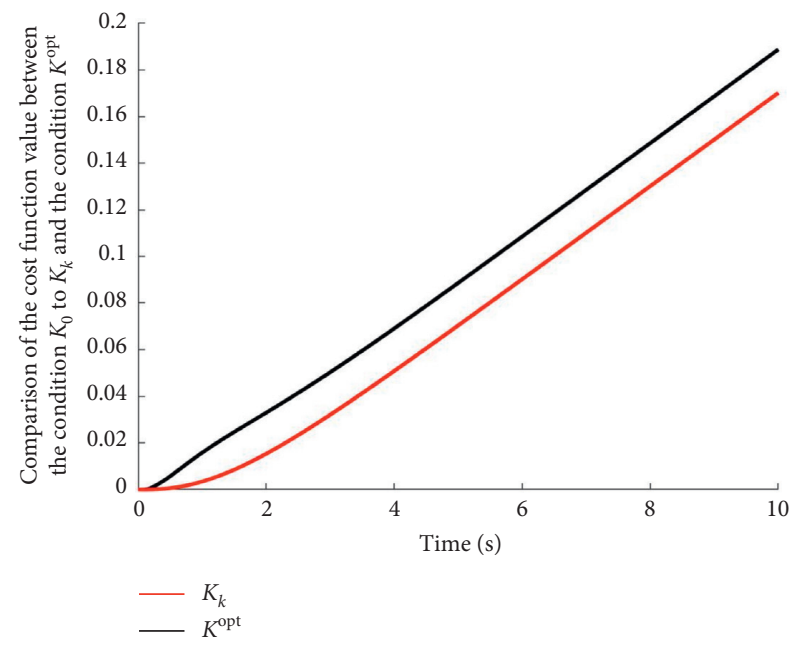

(b)

Figure 10: (a) The comparison of the object deformation information between different $K^{*}$ values and (b) the comparison of the cost between different $K^{*}$ values.

$x_{o}^{\text {opt }}$ is not directly utilized. From $0.15 \mathrm{~s}$ on, the modified deformation trajectory is calculated through

$$
x_{o}=\operatorname{decay} * x_{o}+(1-\text { decay }) * x_{o}^{\mathrm{opt}},
$$

where decay $=e^{\text {rate* }\left(t-t^{\prime}\right)}$ with rate $=-1$ and $t^{\prime}$ being the time when equation (17) is satisfied for the first time, which results in that $x_{o}$ keeps going up before it smoothly converges to $x_{o}^{\text {opt }}$. 


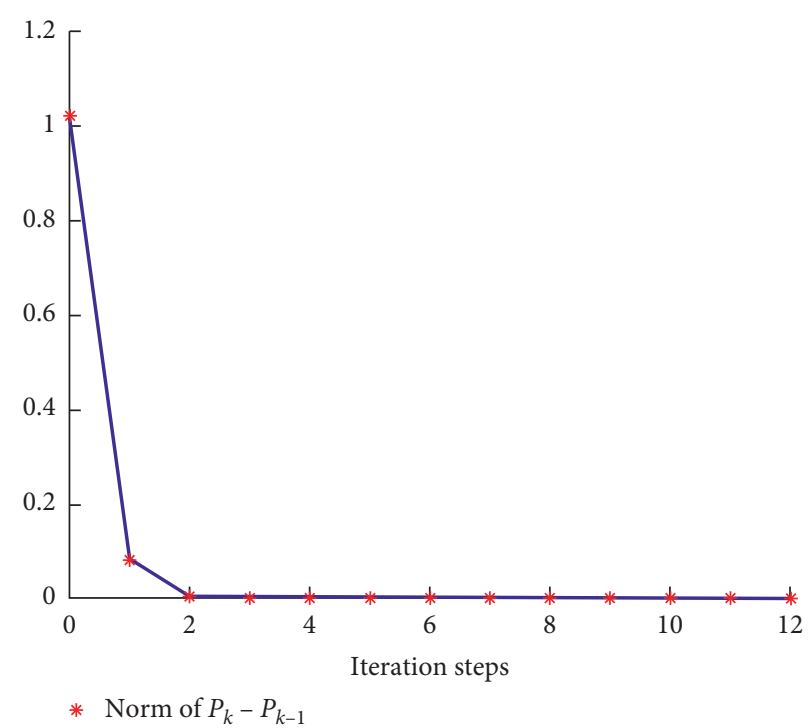

FIgURE 11: Euclidean norm value of $P_{k}-P_{k-1}$.

TABLE 2: $K_{k}$ and $K_{\text {opt }}$ values in the condition with different weights.

\begin{tabular}{lccccc}
\hline Weight $\left[\begin{array}{ll}W_{x} & W_{f}\end{array}\right]$ & {$[1,1]$} & {$[1,2]$} & {$[2,1]$} & {$[3,1]$} & {$[1,3]$} \\
\hline$K_{k}$ & {$[-0.2780,0.0901]$} & {$[-0.1464,0.0481]$} & {$[-0.5117,0.1620]$} & {$[-0.7187,0.2231]$} & {$[-0.0995,0.0329]$} \\
$K_{\text {opt }}$ & {$[-0.2361,0.0890]$} & {$[-0.1213,0.0469]$} & {$[-0.4495,0.1626]$} & {$[-0.6458,0.2259]$} & {$[-0.0817,0.0319]$} \\
\hline
\end{tabular}

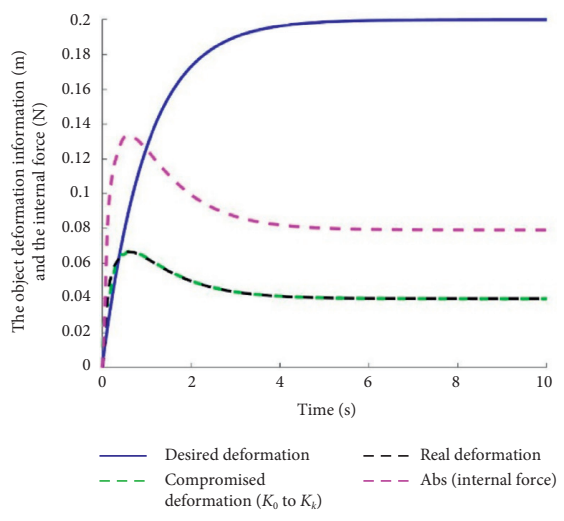

(a)

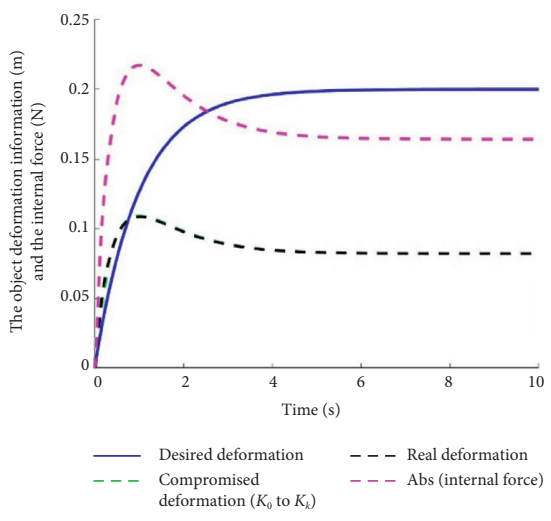

(b)

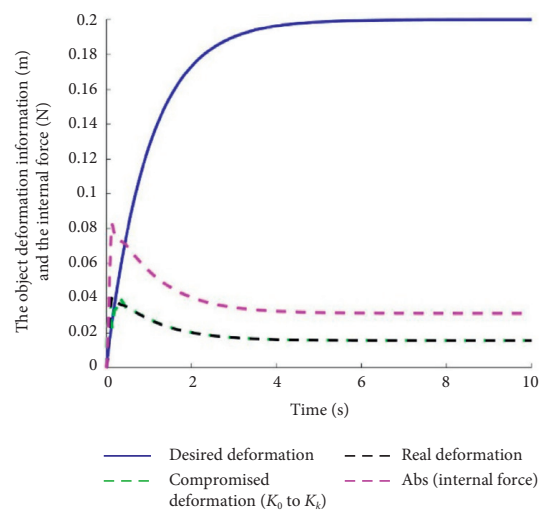

(c)

FIGURE 12: The deformation information and the internal force in the condition with different weights. (a) $\left[W_{x} W_{f}\right]=[1,1]$, (b) $\left[\begin{array}{ll}W_{x} & W_{f}\end{array}\right]=[3,1]$, and (c) $\left[W_{x} W_{f}\right]=[1,3]$.

In Figure 11, we also see a fast converging rate of $P_{k}$ in this case, with the value of $\left\|P_{k}-P_{k-1}\right\|$ decreasing less than $\varepsilon$ within a few steps of iterations.

In a word, this model-free computational adaptive optimal method is feasible to seek a solution to minimize cost function in equation (12) in bimanual roots holdingdamping-spring-model task, minimizing the sum of the tracking position errors and force errors.

Case 4. In this case, we applied the adaptive optimal method under different conditions to test its adaptation performance. The object impedance model is set as $-f_{o}=0.01 \dot{x}_{o}+$ $2 x_{0}$ in this case.
Different weight values and the corresponding $K_{k}$ and the $K_{\text {opt }}$ values are shown in Table 2, and the corresponding simulation results are shown in Figure 12. Through these results, on the one hand, this method is proved to be compatible in different conditions. As we can see from Table 2, all $K_{k}$ are closed to $K_{\text {opt }}$ in different weight conditions. Besides, if we notice the difference between the object impedance model in Case 4 and that in Case 3, we can conclude that this algorithm is also compatible in dealing with different kinds of damping-spring models since the differences between $K_{k}$ and $K_{\mathrm{opt}}$ among all the presented results are small. On the other hand, the practicality of this method is also validated. Figure 12 reveals how the weights 
work: if we want to keep the position tracking errors smaller, we can increase the value of $W_{x}$; vice verses, if we want to keep the force tracking to more precise, we can increase the value of $W_{f}$. For instance, comparing Figures 12(a) and $12(\mathrm{c})$, as the desired holding force is 0 , the increase of $W_{f}$ (from 1 to 3 ) decreases the force tracking errors, in spite of losing some precision in the position tracking.

\section{Conclusions}

This paper introduces an impedance model-based optimal approach to regulate force and position tracking in bimanual robots holding-object task. In the whole system, several algorithms are employed, for example, the CLIK technique to help derived joint positions for robots to move to the right poses according to the desired deformation of the object; the force observer scheme based on generalized momentum theory to help robots to sense the subjected force exerted by the deformed object, instead of installing sensors on EE, such that the robot joint control torques can be correctly compensated and the EE steady-state tracking errors are tackled; and the model-free computational adaptive optimal method to seek a solution to the optimal trade-off between the position and the force tracking errors. The simulation results demonstrate both the effectiveness and the practicality of this optimal scheme, in which the $K_{k}$ value in equation (21) is acquired within a very short time without knowing the object's impedance model, which is also very closed to the exact optimal value $K_{\mathrm{opt}}$. And through modifying the weight value in the cost function, different desired control performance can be easily achieved. In a word, by properly integrating these techniques, the bimanual robots can "feel" the force raised by the held object and exert the optimal regulation on their poses according to user-defined weights.

\section{Data Availability}

No data were used to support this study.

\section{Conflicts of Interest}

The authors declare that there are no conflicts of interest regarding the publication of this paper.

\section{Acknowledgments}

This work was supported in part by the Engineering and Physical Sciences Research Council (EPSRC) under Grant EP/S001913. Thanks are due to Dr. Yanan Li, Mr. Dianye Huang, Mr. Guangzhu Peng, and Dr. Jing Luo for their kind technical support.

\section{References}

[1] J. Zhang, M. Li, Y. Feng, and C. Yang, "Robotic grasp detection based on image processing and random forest," Multimedia Tools and Applications, vol. 79, no. 3-4, pp. 2427-2446, 2020.
[2] J. Zhao, J. Liang, and O. Kroemer, "Towards precise robotic grasping by probabilistic post-grasp displacement estimation," 2019, https://arxiv.org/abs/1909.02129.

[3] J. M. Romano, K. Hsiao, G. Niemeyer, S. Chitta, and K. J. Kuchenbecker, "Human-inspired robotic grasp control with tactile sensing," IEEE Transactions on Robotics, vol. 27, no. 6, pp. 1067-1079, 2011.

[4] M. Kaboli, K. Yao, and G. Cheng, "Tactile-based manipulation of deformable objects with dynamic center of mass," in Proceedings of the 2016 IEEE-RAS 16th International Conference on Humanoid Robots (Humanoids), pp. 752-757, IEEE, Cancun, Mexico, November 2016.

[5] J. Maitin-Shepard, M. Cusumano-Towner, J. Lei, and P. Abbeel, "Cloth grasp point detection based on multipleview geometric cues with application to robotic towel folding," in Proceedings of the 2010 IEEE International Conference on Robotics and Automation, pp. 2308-2315, IEEE, Anchorage, AK, USA, May 2010.

[6] K. Chatzilygeroudis, B. Fichera, I. Lauzana et al., "Benchmark for bimanual robotic manipulation of semi-deformable objects," IEEE Robotics and Automation Letters, vol. 5, no. 2, pp. 2443-2450, 2020.

[7] P. Sapaty, "Military robotics: latest trends and spatial grasp solutions," International Journal of Advanced Research in Artificial Intelligence, vol. 4, no. 4, pp. 9-18, 2015.

[8] A. Gautam and S. Mohan, "A review of research in multirobot systems," in Proceedings of the 2012 IEEE 7th International Conference on Industrial and Information Systems (ICIIS), pp. 1-5, IEEE, Chennai, India, August 2012.

[9] L. Xiao, J. Gong, and J. Chen, "Industrial robot control systems: a review," in Proceedings of the 11th International Conference on Modelling, Identification and Control (ICMIC2019), pp. 1069-1082, Springer, Singapore, 2020.

[10] Y. Gan, J. Duan, M. Chen, and X. Dai, "Multi-robot trajectory planning and position/force coordination control in complex welding tasks," Applied Sciences, vol. 9, no. 5, p. 924, 2019.

[11] J. A. Marvel, R. Bostelman, and J. Falco, "Multi-robot assembly strategies and metrics," ACM Computing Surveys, vol. 51, no. 1, pp. 1-32, 2018.

[12] L. Briñón-Arranz, A. Renzaglia, and L. Schenato, "Multirobot symmetric formations for gradient and hessian estimation with application to source seeking," IEEE Transactions on Robotics, vol. 35, no. 3, pp. 782-789, 2019.

[13] C. Yang, Y. Jiang, Z. Li, W. He, and C.-Y. Su, "Neural control of bimanual robots with guaranteed global stability and motion precision," IEEE Transactions on Industrial Informatics, vol. 13, no. 3, pp. 1162-1171, 2017.

[14] J. Azeta, C. Bolu, A. A. Abioye et al., "A review on humanoid robotics in healthcare," in Proceedings of the 4th International Conference on Mechatronics and Mechanical Engineering, Kuala Lumpur, Malaysia, November 2017.

[15] H. K. Kalra and R. Chadha, "A review study on humanoid robot SOPHIA based on artificial intelligence," International Journal of Technology and Computing (IJTC). Techlive Solutions, vol. 4, no. 3, 2018.

[16] Z. G. Hou, L. Cheng, and M. Tan, "Multicriteria optimization for coordination of redundant robots using a dual neural network," IEEE Transactions on Systems, Man, and Cybernetics, Part B (Cybernetics), vol. 40, no. 4, pp. 1075-1087, 2009.

[17] Z. G. Hou, L. Cheng, and M. Tan, "Decentralized robust adaptive control for the multiagent system consensus problem using neural networks," IEEE Transactions on Systems, Man, and Cybernetics, Part B (Cybernetics), vol. 39, no. 3, pp. 636-647, 2009. 
[18] Y. Jiang, Z. Liu, C. Chen, and Y. Zhang, "Adaptive robust fuzzy control for dual arm robot with unknown input deadzone nonlinearity," Nonlinear Dynamics, vol. 81, no. 3, pp. 1301-1314, 2015.

[19] Z. Lu, P. Huang, and Z. Liu, "Relative impedance-based internal force control for bimanual robot teleoperation with varying time delay," IEEE Transactions on Industrial Electronics, vol. 67, no. 1, pp. 778-789, 2020.

[20] D. Sun, Q. Liao, and A. Loutfi, "Single master bimanual teleoperation system with efficient regulation," IEEE Transactions on Robotics, vol. 36, no. 4, pp. 1022-1037, 2020.

[21] F. Zhang, J. Qu, H. Liu, and Y. Fu, "A pose/force symmetric coordination method for a redundant dual-arm robot," Assembly Automation, vol. 38, no. 5, pp. 678-688, 2018.

[22] Z. Lu, P. Huang, Z. Liu, and H. Chen, "Fuzzy-observer-based hybrid force/position control design for a multiple-samplingrate bimanual teleoperation system," IEEE Transactions on Fuzzy Systems, vol. 27, no. 7, pp. 1383-1396, 2018.

[23] H. Qiao, M. Wang, J. Su et al., "The concept of "attractive region in environment" and its application in high-precision tasks with low-precision systems," IEEE/ASME Transactions on Mechatronics, vol. 20, no. 5, pp. 2311-2327, 2014.

[24] M. Matinfar and K. Hashtrudi-Zaad, "Optimization-based robot impedance controller design," in Proceedings of the 2004 43rd IEEE Conference On Decision And Control (CDC) (IEEE cat. No. 04CH37601), pp. 1246-1251, IEEE, Nassau, Bahamas, December 2004.

[25] M. Matinfar and K. Hashtrudi-Zaad, "Optimization-based robot compliance control: geometric and linear quadratic approaches," The International Journal of Robotics Research, vol. 24, no. 8, pp. 645-656, 2005.

[26] D. Wang, D. Liu, Q. Wei, D. Zhao, and N. Jin, "Optimal control of unknown nonaffine nonlinear discrete-time systems based on adaptive dynamic programming," Automatica, vol. 48, no. 8, pp. 1825-1832, 2012.

[27] R. Johansson and M. W. Spong, "Quadratic optimization of impedance control," in Proceedings of the 1994 IEEE International Conference on Robotics and Automation, pp. 616621, IEEE, San Diego, CA, USA, May 1994.

[28] D. Liu, D. Wang, F.-Y. Wang, H. Li, and X. Yang, "Neuralnetwork-based online HJB solution for optimal robust guaranteed cost control of continuous-time uncertain nonlinear systems," IEEE Transactions on Cybernetics, vol. 44, no. 12, pp. 2834-2847, 2014.

[29] Y. Jiang and Z.-P. Jiang, "Computational adaptive optimal control for continuous-time linear systems with completely unknown dynamics," Automatica, vol. 48, no. 10, pp. 2699-2704, 2012.

[30] S. S. Ge, Y. Li, and C. Wang, "Impedance adaptation for optimal robot-environment interaction," International Journal of Control, vol. 87, no. 2, pp. 249-263, 2014.

[31] A. Stolt, "On robotic assembly using contact force control and estimation," Ph.D. thesis, Department of Automatic Control, Lund University, Lund, Sweden, 2015.

[32] A. De Luca and R. Mattone, "Actuator failure detection and isolation using generalized momenta," in Proceedings of the 2003 IEEE International Conference on Robotics and Automation (Cat. No. 03CH37422), pp. 634-639, IEEE, Taipei, Taiwan, September 2003.

[33] G. Peng, C. Yang, W. He, and C. L. P. Chen, "Force sensorless admittance control with neural learning for robots with actuator saturation," IEEE Transactions on Industrial Electronics, vol. 67, no. 4, pp. 3138-3148, 2019.
[34] C. Yang, G. Peng, Y. Li et al., "Neural networks enhanced adaptive admittance control of optimized robot-environment interaction," IEEE Transactions on Cybernetics, vol. 49, no. 7, pp. 2568-2579, 2019.

[35] A. Wahrburg, B. Matthias, and H. Ding, "Cartesian contact force estimation for robotic manipulators-a fault isolation perspective," IFAC-PapersOnLine, vol. 48, no. 21, pp. 1232-1237, 2015.

[36] A. Wahrburg, E. Morara, G. Cesari et al., "Cartesian contact force estimation for robotic manipulators using Kalman filters and the generalized momentum," in Proceedings of the 2015 IEEE International Conference on Automation Science and Engineering (CASE), pp. 1230-1235, IEEE, Gothenburg, Sweden, August 2015.

[37] B. Siciliano, "A closed-loop inverse kinematic scheme for online joint-based robot control," Robotica, vol. 8, no. 3, pp. 231-243, 1990.

[38] P. Chiacchio, S. Chiaverini, L. Sciavicco, and B. Siciliano, "Closed-loop inverse kinematics schemes for constrained redundant manipulators with task space augmentation and task priority strategy," The International Journal of Robotics Research, vol. 10, no. 4, pp. 410-425, 1991.

[39] P. Falco and C. Natale, "On the stability of closed-loop inverse kinematics algorithms for redundant robots," IEEE Transactions on Robotics, vol. 27, no. 4, pp. 780-784, 2011.

[40] E. Bjoerlykhaug, "A closed loop inverse kinematics solver intended for offline calculation optimized with GA," Robotics, vol. 7, no. 1, p. 7, 2018.

[41] F. L. Lewis, D. Vrabie, and V. L. Syrmos, Optimal Control, Wiley, Hoboken, NJ, USA, 1995.

[42] B. Siciliano and O. Khatib, Springer Handbook of Robotics, Springer, Cham, Switzerland, 2016.

[43] J. J. Craig, Introduction to Robotics: Mechanics and Control, Pearson/Prentice Hall, London, UK, 2009. 